\title{
Simulação do saldo de radiação na Serra da Mantiqueira
}

\author{
Pabricio M. O. Lopes' ${ }^{1}$, Dalton M. Valeriano ${ }^{2}$, Bernardo B. da Silva ${ }^{3}$, \\ Geber B. de A. Moura ${ }^{1}$ \& Alexsandro O. da Silva ${ }^{4}$
}

\begin{abstract}
RESUMO
A influência do desmatamento da Mata Atlântica sobre o microclima da Serra da Mantiqueira ainda não é totalmente compreendida. Para conhecer as consequências do desmatamento sobre o clima serrano é necessário realizar estudos sobre o balanço de radiação na superfície. A falta de dados possibilita conjugar imagens de satélite com dados meteorológicos em um Sistema de Informação Geográfica na determinação do balanço de radiação. O presente estudo teve por objetivo avaliar o modelo MTCLIM em dias de céu claro ou nublado para simular o balanço de radiação na Serra da Mantiqueira, divisa entre os estados de São Paulo, Minas Gerais e Rio de Janeiro, Brasil. Imagens diárias, semanais e dezesseis dias do sensor MODIS disponíveis em 2003 foram utilizadas em rotinas específicas do MTCLIM. Alvos específicos foram selecionados para avaliar o comportamento do balanço de radiação. Observou-se que o balanço de radiação acompanhou a topografia local e é influenciado pelo tipo de uso da terra. Conclui-se que a temperatura da superfície contribui para aumentar a temperatura do ar implicando em diminuição do balanço de radiação sobre pastagem. O modelo MTCLIM demonstrou boa correlação para a temperatura do $\operatorname{ar}\left(\mathrm{R}^{2}=0,82\right)$ e para a radiação solar global $\left(R^{2}=0,71\right)$.
\end{abstract}

Palavras-chave: modis, desmatamento, MTCLIM, krigagem

\section{Simulation of net radiation in the Mantiqueira mountain}

\begin{abstract}
The influence of deforestation of the Atlantic Forest on the microclimate of the mountain Mantiqueira is not yet fully understood. To understand the consequences of deforestation on the highland climate research is needed about the surface radiation balance. The lack of data allows combining satellite images with meteorological data in a Geographic Information System in determining the radiation balance. The study aimed to evaluate the MTCLIM model in cloudless days or cloudy sky and simulate the radiation balance in the Mantiqueira mountain, between São Paulo, Minas Gerais and Rio de Janeiro, Brazil. Daily images, weekly and sixteen days MODIS available in 2003 were used in specific routines from MTCLIM. Specific targets were selected to evaluate the behavior of the radiation balance. It was observed that the radiation balance followed the local topography and is influenced by the type of land use. It follows that the surface temperature contributes to elevate the air temperature, resulting in lesser radiation balance on pasture. The MTCLIM model showed good correlation for air temperature $\left(R^{2}=0.82\right)$ and solar radiation $\left(R^{2}=0.71\right)$.
\end{abstract}

Key words: modis, deforestation, MTCLIM, kriging 


\section{INTRODUÇÃO}

Originalmente, a Mata Atlântica se estendia por todo o litoral brasileiro ocupando uma área de $1,3 \times 10^{6} \mathrm{~km}^{2}$. Esta área foi reduzida a aproximadamente $100 \mathrm{mil} \mathrm{km}^{2}$, o que equivale a 7,6\% de sua extensão original (Morellato \& Haddad, 2000). Porém ainda restam fragmentos de floresta na Serra da Mantiqueira, no sudeste do Brasil. Deste fato resulta a importância de se realizar estudos ambientais em toda a extensão da mata serrana para que seja possível conhecer melhor o complexo funcionamento deste bioma, visando a um desenvolvimento sustentável (São Thiago et al., 2007). Para compreender a influência do desmatamento sobre o microclima serrano é extremamente importante estudar o balanço de radiação à superfície $(\mathrm{Rn})$. Além disso, Rn pode ser usado para inferir quanto a prováveis mudanças climáticas sobre a serra e também se os regimes evapotranspirativos dos ecossistemas terrestres estão sofrendo alterações (Allen et al., 2007). A variabilidade espacial e temporal de Rn também é útil para previsão de tempo, a modelagem hidrológica e a agrometeorologia (Di Pace et al., 2008).

A medida de Rn não é rotineira em estações meteorológicas uma vez que depende muito da natureza da superfície e também da geometria do terreno (Oliphant et al., 2003); portanto, sofre grande influência do albedo e temperatura da superfície (Silva et al., 2011). Por outro lado, o custo operacional, a manutenção e a calibração dos saldos radiômetros são elevados justificando-se seu uso somente por meio de experimentos de campo através dos quais que se visa obter as componentes do balanço de energia (Silva et al., 2007; Fietz \& Fisch, 2009). Uma alternativa de baixo custo operacional para estimar Rn em uma área extensa, heterogênea e de topografia acidentada, é integrar dados de satélite a modelos micrometeorológicos em um sistema de informação geográfica (Lopes, 2006). A utilidade operacional dessa agregação depende das formulações empregadas, da qualidade e da disponibilidade dos dados meteorológicos e orbitais. Borges et al. (2010) atentam para o fato de que os modelos são, em geral, restritos às condições para as quais foram desenvolvidos apresentando problemas de transferibilidade, quando não devidamente calibrados.

Os modelos empregados para estimar Rn em área de topografia acidentada requerem, basicamente, dados de temperatura da superfície e do ar, albedo e emissividade, radiação solar global e um Modelo de Elevação Digital (MED). A temperatura do ar pode ser estimada facilmente utilizando-se um MED fornecido por satélites artificiais (Cavalcanti et al., 2006; Lopes et al., 2011). O albedo e a temperatura da superfície são fornecidos dos produtos dos satélites Terra e Aqua do sensor MODIS (Xiong et al., 2009) ou calculados de cenas do Landsat (Silva et al., 2005; Di Pace et al., 2008). A radiação solar global pode ser estimada pontualmente (Fietz \& Fisch, 2009) ou por meio de imagens de satélite utilizando-se algoritmos, como os descritos por Santos \& Silva (2010) e Allen et al. (2007). A exposição, a inclinação e o fator de visada do terreno são calculados do MED (Lopes, 2006). Deste modo, um modelo que leva em conta os efeitos do relevo sobre a temperatura e a radiação solar global é o MTCLIM (Simulador de Microclima montanhoso). O modelo MTCLIM infere na temperatura do ar e radiação global em locais de morro, de serra ou de montanhas (Hungerford et al., 1989; Glassy \& Running, 1994).
O modelo MTCLIM foi aplicado e avaliado, inicialmente, por Running et al. (1987) em seis locais separados $90 \mathrm{~km}$ ao redor do posto meteorológico do aeroporto de Missoula, Moutana (EUA) demonstrado boas correlações para a temperatura do ar e radiação global mas que deveriam ser feitas correções no gradiente vertical de temperatura. Hungerford et al. (1989) corrigiram este erro e aplicar o MTCLM também em Montana, obtendo boas estimativas de temperatura do ar e radiação global. Novas adaptações no modelo foram feitas para simular a temperatura do ar e a radiação global em regiões de climas árido (Thornton et al., 2000) e de clima tropical (Thornton \& Running, 1999). No Brasil, o modelo MTCLIM tem sido avaliado e endereçado a fornecer as entradas em modelos de processos fisiológicos (Almeida \& Landsberg, 2003) em ecossistemas florestais.

A utilização conjunta de imagem de satélite, de dados meteorológicos e de saídas de modelo climático em um Sistema de Informação Geográfica (SIG) permitiu que Gomes et al. (2009) obtivessem Rn em áreas de cana-de-açúcar e cerrado brasileiro enquanto Di Pace et al. (2008) avaliaram o impacto da topografia no cômputo de Rn. Silva et al. (2011) estimaram $\mathrm{Rn}$ e analisaram as implicações climáticas locais decorrentes das áreas irrigadas.

Assim, este trabalho tem por objetivo avaliar a temperatura do ar e a radiação solar global obtidas pelo modelo MTCLIM em dias de céu claro ou nublado e simular o balanço de radiação numa porção da Serra da Mantiqueira, divisa entre os estados de Minas Gerais, São Paulo e Rio de Janeiro, Brasil. Buscase, ainda, inferir sobre as mudanças no balanço de radiação devido aos regimes climáticos, a topografia e ao uso da terra no ano de 2003.

\section{Material e Métodos}

Aárea de estudo abrange uma porção da Serra da Mantiqueira e do Vale do Rio Paraíba do Sul $\left(15 \times 25 \mathrm{~km}^{2}\right)$ contendo o Parque Nacional do Itatiaia, divisa entre os estados de Minas Gerais, São Paulo e Rio de Janeiro. As precipitações pluviais anuais variam entre 1.000 a $2.000 \mathrm{~mm}$ na serra e entre 1.000 a 1.600 $\mathrm{mm}$ no vale. $\mathrm{O}$ clima serrano apresenta invernos frios e verãos brandos, com temperaturas médias anuais variando de 18 a $19^{\circ} \mathrm{C}$ e umidade relativa do ar maior que $70 \%$. O uso da terra é classificado principalmente como floresta primária até a cota de $1.800 \mathrm{~m}$, pastagem de altitude em cotas superiores e áreas de reflorestamento. Verificam-se, também, áreas antrópicas, tanto na serra quanto no vale, Figura 1 . A área teste está marcada por um retângulo em preto.

A radiação solar global diária $\left(\mathrm{Rs} \mathrm{MJ} \mathrm{m}^{-2}\right)$, as temperaturas máximas do ar $\left(\mathrm{Tx},{ }^{\circ} \mathrm{C}\right)$ e a mínima $\left(\mathrm{T} n,{ }^{\circ} \mathrm{C}\right)$ foram obtidas por sensores instalados em plataformas de coleta de dados (PCD) localizada no município de Queluz (22 ${ }^{\circ} 32^{\prime}$ S; $44^{\circ}$ 46’ W; 564 $\mathrm{m})$, São Paulo, fornecidos pelo Centro de Previsão do Tempo e Estudos Climáticos (CPTEC) referentes ao ano de 2003. O modelo MTCLIM foi validado usando-se medidas de Tx e Tn feitas no local da PCD de Cachoeira Paulista $\left(22^{\circ} 40^{\prime} \mathrm{S}, 45^{\circ} 00^{\prime}\right.$ W; $563 \mathrm{~m}$ ), SP extrapolado-as para o local da PCD de Queluz para o ano de 2003. Os valores estimados de t e Rs foram correlacionados com os medidos no local da PCD de Queluz, pela análise de regressão para o ano de 2003. 


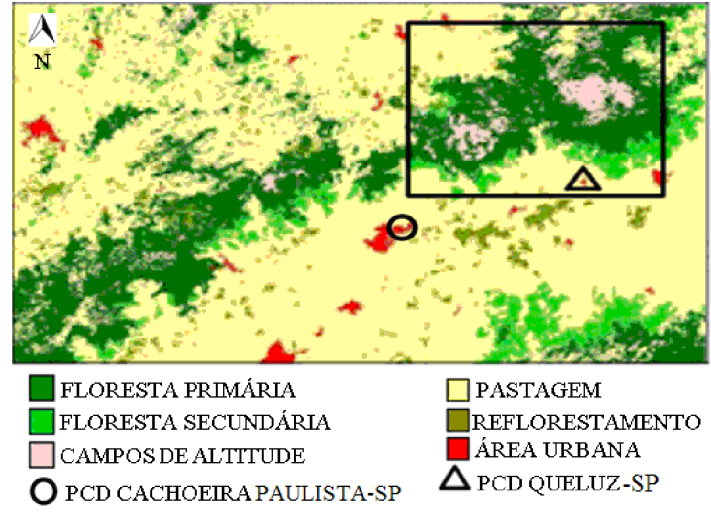

Figura 1. Classificação não supervisionada do uso da terra de uma porção da Serra da Mantiqueira e do Vale do Rio Paraíba do Sul, localizada entre $22^{\circ} 33^{\prime}$ $\mathrm{S}$ e $45^{\circ} 30^{\prime} \mathrm{W} ; 22^{\circ} 30^{\prime} \mathrm{S}$ e $44^{\circ} 30^{\prime} \mathrm{W}$

As estimativas da temperatura do ar $\left(\mathrm{t},{ }^{\circ} \mathrm{C}\right)$ e $\mathrm{Rs}$ foram feitas usando-se os dados da PCD de Queluz como entrada no modelo MTCLIM para inferir dados pontualmente em 349 pontos escolhidos aleatoriamente na área do Parque Nacional do Itatiaia (Figura 1). Este modelo infere dados t, Tx, Tn e Rs medidos por sensores instalados em uma estação meteorológica automática localizada no vale para um local de interesse sobre a serra (Hungerford et al., 1989). Os dados meteorológicos fornecidos pela estação são: Tx, Tn e precipitação diária. Do ponto de interesse, onde se deseja inferir os dados, são necessários: as coordenadas geográficas, a declividade, o aspecto, os ângulos de elevação horizontais leste-oeste e o gradiente vertical de temperatura em que, neste trabalho, foi usado o valor de $-0,0065^{\circ} \mathrm{C} \mathrm{m}^{-1}$.

Inicialmente, assume-se, no modelo MTCLIM que a função horária da temperatura diária do ar (t) tem forma senoidal. A t do local de interesse é calculada através de Tx e Tn fornecidas pela estação meteorológica localizada no vale. A integração da função seno sobre os três quadrantes produz a Eq. 1 para $t$ do local de interesse, proposta por Hungerford et al. (1989):

$$
\begin{aligned}
t & =0,212 \times\left[\left(\mathrm{Tx}_{\text {med }}-\mathrm{Tx}_{\text {est }}\right) \times \tau+\mathrm{Tx}_{\text {est }}-\left(\frac{\mathrm{Tx}_{\text {est }}+\mathrm{Tn}_{\text {est }}}{2}\right)\right]+ \\
& +\frac{\mathrm{Tx}_{\text {est }}+\mathrm{Tn}_{\text {est }}}{2}
\end{aligned}
$$

em que:

$\mathrm{Tx}_{\text {est }}, \mathrm{Tx}_{\text {med }}, \mathrm{Tn}_{\text {est }}$ - temperaturas máxima, mínima diárias do ar, estimadas (est) e medidas (med) para um ponto na serra, respectivamente, ${ }^{\circ} \mathrm{C}$

$\tau$ - transmitância atmosférica diária

A constante 0,212 é um coeficiente empírico de ajuste; a Eq. 2 é usada para estimar a $\mathrm{R}_{\downarrow \mathrm{s}}\left(\mathrm{W} \mathrm{m}^{-2}\right)$ expressa por Hungerford et al. (1989) e Glassy \& Running (1994) como sendo:

$$
\begin{aligned}
R_{\downarrow S} & =I_{s}+D_{s}=S_{O} \times N \times \tau^{\mathrm{AM}} \times \cos \varphi+\cos ^{2}\left(\frac{\beta}{2}\right) \times \\
& \times\left\{\left[\left(S_{0} N \cos Z\right)^{2} \tau^{\mathrm{AM}}\right]^{0,5}\left(1-\mathrm{S}_{\mathrm{O}} \mathrm{N} \tau^{\mathrm{AM}} \cos \mathrm{Z}\right)^{0,5}\right\}
\end{aligned}
$$

em que:

$\mathrm{I}_{\mathrm{s}} \quad$ - radiação solar direta, $\mathrm{W} \mathrm{m}^{-2}$

$\beta \quad$ - ângulo de inclinação do terreno, graus

$\mathrm{D}_{\mathrm{s}}$ - radiação difusa à superfície, $\mathrm{W} \mathrm{m}^{-2}$

$\mathrm{S}_{\mathrm{o}} \quad$ - constante solar, $1.367 \mathrm{~W} \mathrm{~m}^{-2}$

$\mathrm{N}$ - fotoperíodo, $\mathrm{h}$

Z - ângulo zenital solar, graus

$\varphi \quad$ - ângulo de incidência entre a normal a uma encosta e os raios solares, graus

AM - massa de ar óptica, calculada usando-se o modelo de Bristow \& Campbell (1984) descrito pelas Eqs. 3, 4 e 5:

$$
\begin{gathered}
\tau=\mathrm{A}\left[1-\exp ^{\left(-0,003 \Delta \mathrm{T}^{2,4}\right)}\right] \\
\mathrm{AM}=\left[\frac{1}{\cos \mathrm{Z}}\right]+1 \times 10^{-7} \\
\Delta \mathrm{T}=\mathrm{T}_{\mathrm{X}_{\mathrm{i}}}-\frac{\left(\mathrm{T}_{\mathrm{n}_{\mathrm{i}}}+\mathrm{T}_{\mathrm{x}_{\mathrm{i}+1}}\right)}{2}
\end{gathered}
$$

em que:

A - transmitância máxima de céu claro: 0,6 a nível médio do mar, aumentando $0,008 \mathrm{~m}^{-1}$ com a elevação

$\Delta \mathrm{T}$ - intervalo diário de temperatura, ${ }^{\circ} \mathrm{C}$

$\mathrm{Tx}_{\mathrm{i}}, \mathrm{Tx}_{(\mathrm{i}+1)}$ e $\mathrm{Tn}_{\mathrm{i}}$ - temperaturas máxima e mínima para o dia i, ${ }^{\circ} \mathrm{C}$

As médias diárias de Tx, Tn e precipitação do ano 2003 medidas na PCD de Queluz (SP) foram usadas como entrada no modelo MTCLIM para inferir dados de te Rs em 349 pontos distribuídos sobre a Serra da Mantiqueira; em seguida, mensais de t e Rs para cada ponto foram extraídas para a produção de cartas mensais do saldo de radiação solar. O saldo de radiação à superfície diário ( $\left.\mathrm{Rn}, \mathrm{MJ} \mathrm{m}^{-2}\right)$ representa a contabilidade entre os fluxos radiativos de ondas curtas e de ondas longas ascendentes e descentes que atuam no sistema Terra-atmosfera e pode ser estimado pela Eq. 6 (Allen et al., 2002; Silva et al., 2011):

$$
\mathrm{Rn}=\mathrm{R}_{\downarrow \mathrm{S}} \times(1-\alpha)+\mathrm{R}_{\mathrm{L} \downarrow}-\mathrm{R}_{\mathrm{L} \uparrow}-\left(1-\varepsilon_{\mathrm{O}}\right) \mathrm{R}_{\mathrm{L} \downarrow}
$$

em que:

$$
\begin{array}{ll}
\mathrm{R}_{\mathrm{L} \downarrow} & \text { - radiação atmosférica, } M J \mathrm{~m}^{-2} \\
\mathrm{R}_{\mathrm{L} \uparrow} & \text { - radiação da superfície terrestre, } M J \mathrm{~m}^{-2} \\
\alpha & \text { - albedo da superfície } \\
\varepsilon_{\mathrm{o}} & \text { - emissividade da superfície }
\end{array}
$$

Em terrenos montanhosos Rn pode ser expresso combinando a equação proposta por Dozier \& Outcalt (1979) com a Eq. 6 de Allen et al. (2002) dada pela Eq. 7 :

$$
\begin{aligned}
\mathrm{Rn} & =\operatorname{Rs} \times(1-\alpha)+\cos ^{2}\left(\frac{\beta}{2}\right)\left[\left(1-\mathrm{V}_{\mathrm{d}}\right) \times \varepsilon_{\mathrm{a}} \sigma \mathrm{T}_{\mathrm{a}}^{2}+\right. \\
& \left.+\left(\mathrm{V}_{\mathrm{d}}-1\right) \times \varepsilon_{\mathrm{o}} \sigma \mathrm{T}_{\mathrm{s}}^{4}-\left(1-\varepsilon_{\mathrm{o}}\right)\left(1-\mathrm{V}_{\mathrm{d}}\right) \times \varepsilon_{\mathrm{a}} \sigma \mathrm{T}_{\mathrm{a}}^{2}\right]
\end{aligned}
$$

em que: 
$\varepsilon_{\mathrm{a}} \quad$ - emissividade atmosférica

$\sigma^{\mathrm{a}} \quad$ - constante de Stefan-Boltzmann: 5,6697x $\mathrm{N}^{-8} \mathrm{~W} \mathrm{~m}^{-2} \mathrm{~K}^{-4}$

$\mathrm{T}_{\mathrm{a}} \quad$ - temperatura média mensal do ar, $\mathrm{K}$

$\mathrm{T}_{\mathrm{s}} \quad$ - temperatura média da superfície, $\mathrm{K}$

$\mathrm{V}_{\mathrm{d}}$ - fator de visada do céu

$\beta \quad$ - ângulo de inclinação do terreno, graus

$\mathrm{O}$ fator $\mathrm{V}_{\mathrm{d}}$ representa a fração da radiação solar difusa que chega num dado ponto do terreno relativa àquela que atinge uma superfície horizontal não obstruída pela vizinhança do terreno. $\mathrm{O}$ intervalo de variação de $\mathrm{V}_{\mathrm{d}}$ é de 0 (máxima influência do terreno) a 1 (nenhuma influência do terreno). $\mathrm{O} \mathrm{V}_{\mathrm{d}}$ é estimado em função do ângulo do horizonte local em dada direção para cada ponto do MED, podendo ser expresso por meio da Eq. 8 (Dozier \& Outcalt, 1979):

$$
\begin{aligned}
\mathrm{V}_{\mathrm{d}} & \approx \frac{1}{2 \pi} \int_{0}^{2 \pi}\left[\cos \beta \times \operatorname{sen}^{2} \mathrm{H}_{\varphi}+\operatorname{sen} \beta \times \cos (\varphi-\psi) \times\right. \\
& \left.\times\left(\mathrm{H}_{\varphi}-\operatorname{senH}_{\varphi} \times \cos \mathrm{H}_{\varphi}\right)\right] \mathrm{d} \varphi
\end{aligned}
$$

em que:

$\psi \quad$ - ângulo azimutal do terreno, graus

$\mathrm{H} \varphi$ - ângulo horizontal do local, graus

Neste trabalho o $\mathrm{V}_{\mathrm{d}}$ foi simulado utilizando-se o modelo TOPORAD descrito em Lopes (2006).

$O$ albedo da superfície $(\alpha)$ representa o coeficiente de reflexão integrado em todos os comprimentos de onda do espectro de radiação solar. $O$ albedo varia em função da sua cor, das condições de umidade, da densidade de copa, da disposição das folhas e das condições de iluminação. Discriminam-se, em função dessas características, dois tipos de albedo: o albedo "Black Sky" $\left(\alpha_{b S}\right)$ para condições de iluminação direta e o albedo "White Sky" $\left(\alpha_{\mathrm{wS}}\right)$ para condições de iluminação difusa (Lopes, 2006). Desta forma, o albedo é o somatório de $\alpha_{b S}$ e $\alpha_{w s}$ obtidos do produto MOD43B3 do sensor MODIS do satélite Terra, com resolução espacial de $930 \mathrm{~m}$ (LPDAAC, 2012). No entanto, para os propósitos da pesquisa os pixels foram redimensionados para $30 \mathrm{~m}$ de resolução espacial. Este produto é uma composição dos melhores pixels de 16 imagens diárias do sensor MODIS. Neste trabalho foram utilizadas oito composições de 16 dias ao longo do ano de 2003 (Tabela 1).

A emissividade média da superfície $\left(\varepsilon_{0}\right)$ expressa a fração entre o poder emissivo de um corpo real em relação ao de um corpo negro na mesma temperatura e mesmo intervalo de comprimento de onda. A emissividade média foi estimada pelo programa computacional de Valor \& Caselles (1996) dada pelas Eq. (9), (10), (11), (12) e (13). Utilizou-se, como entrada no programa, o produto IVDN (índice de vegetação por diferença normalizada) do sensor MODIS (MOD13Q1) com resolução espacial de $250 \mathrm{~m}$, redimensionadas para $30 \mathrm{~m}$ (Tabela 1) e as emissividades do solo $\left(\varepsilon_{\mathrm{s}}\right)$ de 0,95 e da vegetação $\left(\varepsilon_{\mathrm{v}}\right)$ de 0,98 (Salisbury \& D’ Aria, 1992).

$$
\varepsilon_{\mathrm{o}}=\mathrm{a} \times \mathrm{IVDN}-\mathrm{b}
$$

$$
\begin{gathered}
\mathrm{a}=\frac{\left(\varepsilon_{\mathrm{v}}-\varepsilon_{\mathrm{s}}\right)}{\left(\operatorname{IVDN}_{\mathrm{v}}-\operatorname{IVDN}_{\mathrm{s}}\right)} \\
\mathrm{b}=\frac{\left|\varepsilon_{\mathrm{s}}\left(\mathrm{IVDN}_{\mathrm{v}}-\mathrm{dIVDN}\right)-\varepsilon_{\mathrm{v}}\left(\operatorname{IVDN}_{\mathrm{s}}-\mathrm{dIVDN}\right)\right|}{\left(\mathrm{IVDN}_{\mathrm{v}}-\operatorname{IVDN}_{\mathrm{s}}\right)}+\mathrm{d} \varepsilon \\
\mathrm{IVDN}=\mathrm{IVDN}_{\mathrm{v}} \mathrm{P}_{\mathrm{v}}+\operatorname{IVDN}_{\mathrm{s}}\left(1-\mathrm{P}_{\mathrm{v}}\right)+\mathrm{di} \\
\mathrm{P}_{\mathrm{v}}=\frac{\mathrm{IVDN}-\mathrm{IVDN}_{\min }}{\operatorname{IVDN}_{\text {max }}-\operatorname{IVDN}_{\text {min }}}
\end{gathered}
$$

em que:

$\operatorname{IVDN}_{\mathrm{v}}, \mathrm{IVDV}_{\mathrm{s}}$ - índices das áreas vegetação (v) e de solo desnudo (s), respectivamente

$P_{\mathrm{v}} \quad$ - fração de cobertura vegetal

$\mathrm{IVDN}_{\min } \mathrm{e} \mathrm{IVDN}_{\max }$ - Índices de vegetação mínimo e máximo da imagem de IVDN

di - termo de correção para o IVDN

$\mathrm{d} \varepsilon \quad$ - termo adicional que corrige a reflexão entre as partes diferentes de um sistemas (Valor \& Caselles, 1996)

A emissividade atmosférica é expressa por meio das Eqs. 14 e 15 de acordo com Allen et al. (2002):

$$
\begin{gathered}
\varepsilon_{\mathrm{a}}=0,85 \times\left(-\ln \tau_{\mathrm{ws}}\right)^{0,09} \\
\tau_{\mathrm{ws}}=0,75+2 \times 10^{-5} \mathrm{~h}
\end{gathered}
$$

em que:

$\varepsilon_{a} \quad$ - emissividade atmosférica

$\tau_{\mathrm{ws}} \quad$ - transmitância atmosférica

h - altitude, $\mathrm{m}$

A temperatura média da superfície (Ts) foi obtida dos produtos MODIS (MOD11A1 e MYD11A2) com resolução espacial de $1000 \mathrm{~m}$ para os horários das $10 \mathrm{~h}$ e $30 \mathrm{~min}$ e 13 h $30 \mathrm{~min}$ (hora solar) e reamostrados para $30 \mathrm{~m}$. Os produtos MOD11A1 são imagens diárias obtidas do satélite Terra enquanto os MYD11A2 são imagens semanais produzidas do satélite AQUA. A Tabela 1, seguinte, mostra as principais características dos produtos MODIS utilizadas neste estudo. As imagens MODIS são obtidas gratuitamente na web site do MODIS (Xiong et al., 2009; LPDAAC, 2012). Dos produtos do MODIS foram extraídos 349 pixels distribuídos aleatoriamente sobre a serra para ser usados como entradas no MTCLIM e calcular o balanço de radiação.

As espacializações de $\alpha$, Ts, Rs, t e Rn foram feitas empregando técnica de interpolação por krigeagem ordinária no SURFER Demo 10 (www.goldensoftware.com). Os 349 pixels distribuídos aleatoriamente sobre a área foram obtidos dos produtos do MODIS foram interpolados e sobrepostos com o MDE para representar $\alpha$ e Ts em três dimensões e relacionálos com a classificação do uso da terra (Figura 1). Inicialmente, foi feita uma análise exploratória dos dados para verificação de tendências com gráficos nas direções N-S (latitude) e E-W 
Tabela 1. Produtos MODIS utilizados na simulação de Rn para 2003 em dias de céu aberto

\begin{tabular}{|c|c|c|c|c|c|c|}
\hline $\begin{array}{c}\text { Data das } \\
\text { composições } \\
2003\end{array}$ & $\begin{array}{l}\text { Produtos MODIS } \\
\quad \text { (versão 5) }\end{array}$ & $\begin{array}{c}\text { Satélite } \\
\text { Passagem } \\
\text { (Hora solar) }\end{array}$ & $\begin{array}{c}\text { Resolução } \\
\text { espacial } \\
\text { (reamostrada) }\end{array}$ & $\begin{array}{l}\text { Resolução } \\
\text { radiométrica }\end{array}$ & $\begin{array}{l}\text { Resolução } \\
\text { temporal }\end{array}$ & $\begin{array}{l}\text { Projeção } \\
\text { Datum }\end{array}$ \\
\hline $\begin{array}{l}18 / 02 \text { a } 05 / 03 \\
28 / 07 \text { a } 12 / 09 \\
13 / 07 \text { a } 29 / 09\end{array}$ & $\begin{array}{l}\text { MOD13Q1 } \\
\text { (IVDN) } \\
\text { MOD43B3 } \\
\text { (Albedo) }\end{array}$ & $\begin{array}{c}\text { Terra } \\
10 \text { h } 30 \text { min }\end{array}$ & $250 \mathrm{~m}(30 \mathrm{~m})$ & 16 bits & 16 dias & $\begin{array}{c}\text { Geographic } \\
\text { WGS84 }\end{array}$ \\
\hline $\begin{array}{l}03 / 02 \text { e } 04 / 02 \\
23 / 02 \text { e } 26 / 02 \\
02 / 02 \text { e } 03 / 02 \\
07 / 08 \text { e } 09 / 08 \\
14 / 08 \text { e } 25 / 08 \\
16 / 08 \text { a } 28 / 08\end{array}$ & $\begin{array}{c}\text { MOD11A1 } \\
\text { (Temperatura superfície) }\end{array}$ & $\begin{array}{c}\text { Terra } \\
10 \text { h } 30 \text { min }\end{array}$ & $1000 \mathrm{~m}(30 \mathrm{~m})$ & 16 bits & Diárias & $\begin{array}{c}\text { Geographic } \\
\text { WGS84 }\end{array}$ \\
\hline $\begin{array}{l}02 / 02 \text { a } 09 / 02 \\
27 / 02 \text { a } 05 / 03 \\
05 / 08 \text { a } 12 / 08 \\
13 / 08 \text { a } 20 / 08 \\
29 / 08 \text { a } 05 / 09\end{array}$ & $\begin{array}{c}\text { MYD11A2 } \\
\text { (Temperatura superfície) }\end{array}$ & $\begin{array}{c}\text { Aqua } \\
13 \text { h } 30 \text { min }\end{array}$ & $1000 \mathrm{~m}(30 \mathrm{~m})$ & 16 bits & Oito dias & $\begin{array}{c}\text { Geographic } \\
\text { WGS84 }\end{array}$ \\
\hline
\end{tabular}

(longitude), sendo este estudo fundamental para aplicação das teorias geoestatísticas; em seguida foram calculadas as semivariâncias e testados os modelos: esférico, exponencial e gaussiano, considerando-se a modelagem baseada em apenas uma estrutura; além disto, o variograma experimental foi considerado omnidirecional sendo indiferente à escolha da direção (http://www.rc.unesp.br/igce/aplicada/textodi.html). Neste caso o valor da direção foi 0 e a anisotropia igual a $1 . \mathrm{Na}$ escolha do melhor método e modelo de ajuste foi considerada a "indicação da qualidade de ajuste" (Indicative Goodness of Fit - IGF) fornecida pelo programa computacional Variowin 2.21. O IGF é um número adimensional e quanto mais próximo de zero melhor é o ajuste indicado. $\mathrm{O}$ efeito pepita, o alcance e a contribuição do modelo escolhido foram utilizados como entradas para a interpolação por krigagem de $\alpha$, t, Ts, Rs e Rn. Os modelos utilizados para fevereiro foram o esférico ( $\alpha \mathrm{e}$ ts), modelo gaussiano ( $\mathrm{t}$ e Rs) e modelo exponencial $(\mathrm{Rn})$ e para agosto, o gaussiano ( $\mathrm{t}$ e Rs) e o exponencial (Rn, ts e $\alpha$ ).

Fez-se a avaliação do modelo MTCLIM usando-se o Erro médio (EM) (Eq.16), a Raiz do Erro Quadrático Médio (REQM) (Eq.17), o Coeficientes de precisão (r) e o de correlação $\left(\mathrm{R}^{2}\right)$ :

$$
\begin{gathered}
\mathrm{EM}=\frac{1}{\mathrm{~N}} \sum_{\mathrm{k}=1}^{\mathrm{N}} \mathrm{O}_{\mathrm{i}}-\mathrm{P}_{\mathrm{i}} \\
\mathrm{REQM}=\sqrt{\frac{1}{\mathrm{~N}} \sum_{\mathrm{k}=1}^{\mathrm{N}}\left(\mathrm{O}_{\mathrm{i}}-\mathrm{P}_{\mathrm{i}}\right)^{2}}
\end{gathered}
$$

em que:

$$
\begin{array}{ll}
\mathrm{P}_{\mathrm{i}} & \text { - valores estimados } \\
\mathrm{O}_{\mathrm{i}} & \text { - valores observados } \\
\mathrm{O} & \text { - média dos valores observados } \\
\mathrm{N} & \text { - número de pares de valores estimados e medidos }
\end{array}
$$

\section{Resultados E Discussão}

A Figura 2A e a Figura 2B ilustram os valores diários de Rs e t, inferidos a partir da PCD de Cachoeira Paulista (altitude, $563 \mathrm{~m}$ ) para o local da PCD Queluz (altitude, $564 \mathrm{~m}$ ), SP, no ano de 2003, respectivamente. Na Figura 2A constatou-se que os efeitos do relevo explicam $71 \%$ da estimativa de Rs com REQM de 3,7 $\mathrm{MJ} \mathrm{m}^{-2}$, precisão $(\mathrm{r}=0,84)$ com tendência a superestimar $\left(\mathrm{EM}=+0,58 \mathrm{MJ} \mathrm{m}^{-2}\right)$. No inverno observase tendência de subestimativas de Rs enquanto no verão os erros são menores. Lopes (2006) também constatou tendência semelhante e concluiu que precisam ser feitos ajustes no MTCLIM com relação à cobertura de nuvens. Na Figura 2B a t estimada apresentou boa correlação $\left(\mathrm{R}^{2}=0,82\right)$, RMEQ de $3,7{ }^{\circ} \mathrm{C}$, precisão $(\mathrm{r}=0,90)$ com tendência a superestimar (EM $=+3,32^{\circ} \mathrm{C}$ ). Running et al. (1987) e Hungerford et al. (1989) encontraram valores de $\mathrm{R}^{2}$ para t variando entre 0,88 e 0,94 (Erro padrão $=1,8^{\circ} \mathrm{C}$ ) e $\mathrm{R}^{2}$ para Rs entre 0,38 a 0,55 (Erro padrão $=4,7$ $\mathrm{MJ} \mathrm{m}^{-2}$ ) em seis e 11 locais em Missola, EUA, respectivamente. Em Miami, EUA, Thornthon \& Running (1999) encontraram erros de 3,9 $\mathrm{MJ} \mathrm{m}^{-2}$ e tendência a subestimativas de Rs (EM = $-2,7 \mathrm{MJ} \mathrm{m}^{-2}$ ). De acordo com Hungerford et al. (1989) os erros do modelo MTCLIM podem ser reduzidos caso os dados de entrada sejam de uma estação meteorológica bem próxima do local em que se deseja fazer as simulações, o que reduziria os erros introduzidos pela diferença de cobertura de nuvens sobre a estação meteorológica e o local de interesse. Por sua vez, modelo MTCLIM inicia as estimativas quando o valor de Rs excede $70 \mathrm{~W} \mathrm{~m}^{-2}$ (abertura dos estômatos de árvores coníferas) resultando em diminuição do comprimento do dia de $20 \%$ e, consequentemente, afeta o valor diário de Rs quando comparado com os valores medidos em um PCD.

A Figura 3A e a Figura 3B representam a distribuição espacial do albedo da superfície $(\alpha)$ obtido dos 349 pixels extraídos do produto MOD43 sobre a Serra da Mantiqueira para os meses de fevereiro e agosto de 2003, respectivamente. Observa-se, na Figura 3A, que a altitude varia de 460,4 a 2.555,8 $\mathrm{m}$, sendo $\alpha$ maior nas áreas de altitudes menores, representando $17,4 \%$ da radiação solar incidente enquanto nas áreas de altitudes elevadas o albedo atinge principalmente valores de $8,0 \%$. A diminuição no valor $\alpha$ no lado sul da serra pode estar relacionada, dentre outros fatores, à precipitação pluvial, ao ângulo zenital solar e ao tipo de superfície (Lopes, 2006).

Em fevereiro as precipitações pluviais sobre a área variaram entre 50 a $100 \mathrm{~mm}$ (http://www.cptec.inpe.br/climanalise) fato 
A.

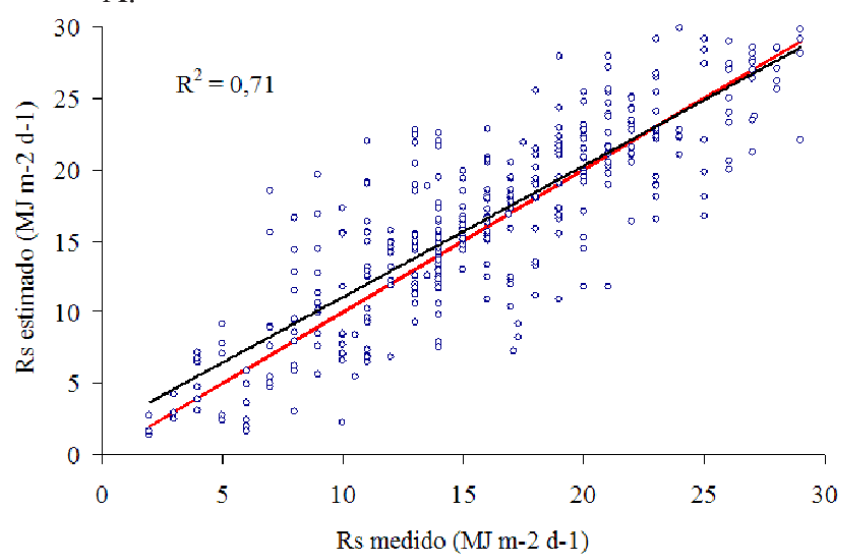

B.

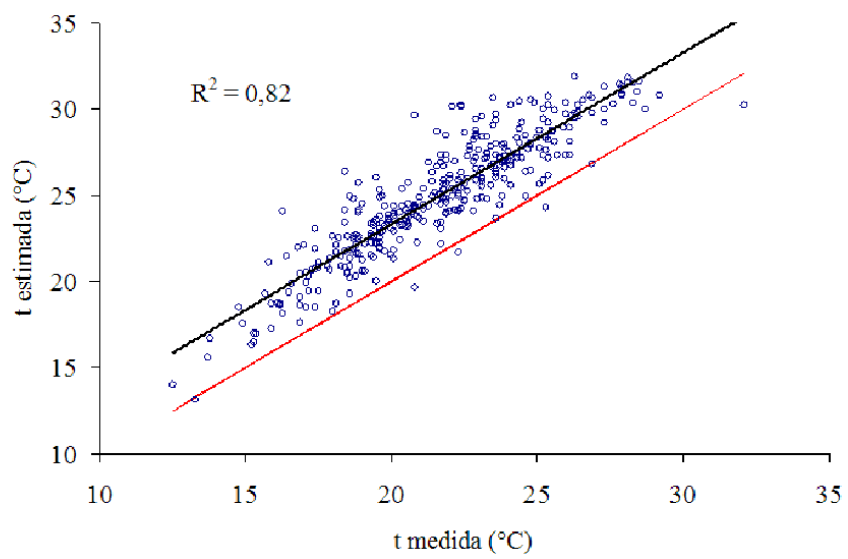

Figura 2. Radiação solar global (A) e temperatura do ar (B) medida e estimada pelo modelo MTCLIM para o local da PCD de Queluz, SP, para os 365 dias do ano de 2003

A.

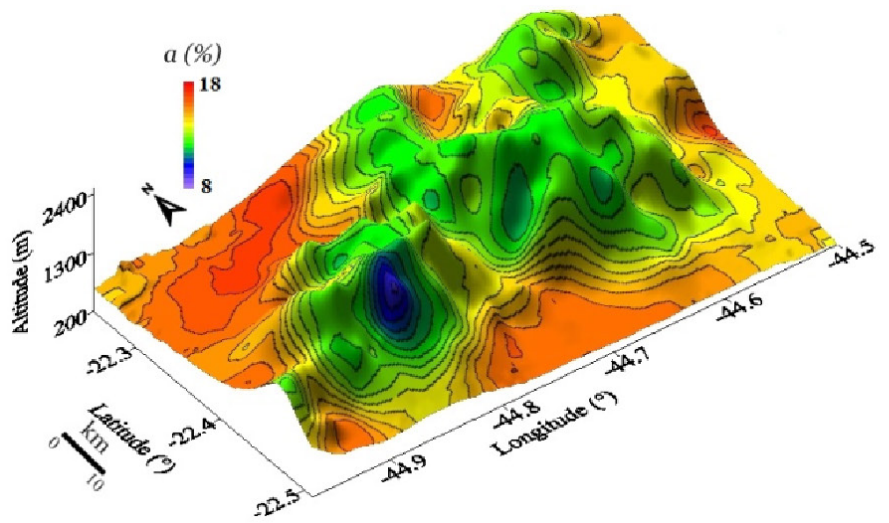

B.

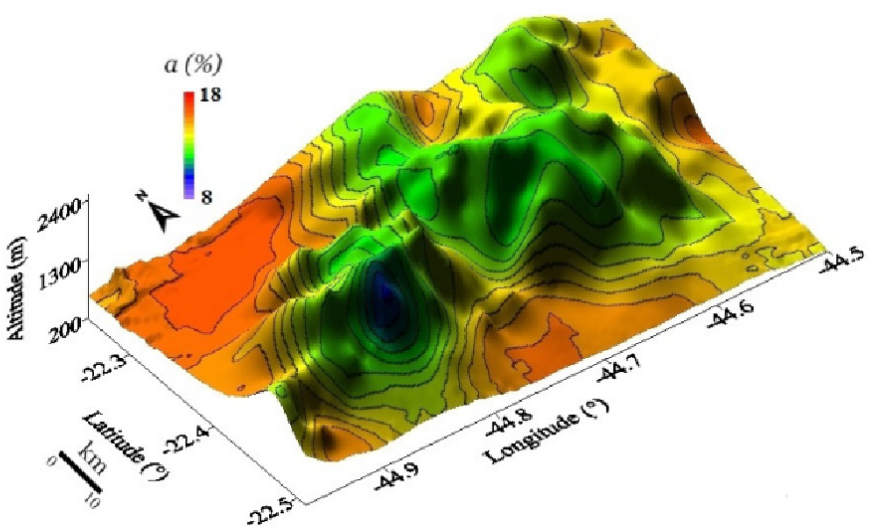

Figura 3. Albedo da superfície para fevereiro (A) e para agosto (B) de 2003 interpolados com 349 pixels extraídos do produto MOD43 às $10 \mathrm{~h} 30 \mathrm{~min}$ (hora solar) sobre a Serra da Mantiqueira

registrado na estação meteorológica automática de Queluz $62,4 \mathrm{~mm}$. A água depositada sobre o solo e a sobre dosseis vegetativos das encostas da serra, diminui o poder de reflexão dos alvos. Silva et al. (2011) observaram que áreas irrigadas sistematicamente apresentaram menor albedo que em solos com baixa cobertura vegetal. Todavia, Oliphant et al. (2003) afirmam que as medidas horárias de $\alpha$ variam fortemente com o ângulo zenital solar enquanto em escala diária $\alpha$ varia em função do tipo de superfície. Na Figura 3B o maior valor do albedo foi estimado nas baixas elevações (pastagens e campos agrícolas) representando $16,9 \%$ da radiação solar incidente e o menor valor de 10,4\% nas altas elevações. Em geral, $\alpha$ de agosto sobre o Parque Nacional do Itatiaia foi maior que o estimado em fevereiro de 2003.

$\mathrm{O}$ aumento em $\alpha$ pode estar relacionado à diminuição das chuvas (em Queluz choveu 36,3 mm em agosto) e à cobertura foliar das plantas. Em geral, o albedo sofreu maior influência da topografia local do que da vegetação, ou seja, à medida em que a elevação aumentou, o albedo também aumentou, concordando com observações feitas por Oliphant et al. (2003) ao estudar os Alpes da Nova Zelândia.

As temperaturas médias da superfície (Ts) interpolada para os 349 pixels extraídos do produto MOD11 sobre a Serra da Mantiqueira para os meses de fevereiro e de agosto de 2003, são ilustradas nas Figuras $4 \mathrm{~A} \mathrm{e} 4 \mathrm{~B}$, respectivamente. Na Figura 4A a Ts variou entre 27,3 a $36,6^{\circ} \mathrm{C}$, com amplitude térmica de $9,3^{\circ} \mathrm{C}$; em geral, as isotermas $\left(1,0^{\circ} \mathrm{C}\right)$ mostram que a Ts diminui com o aumento da altitude apresentado gradientes bastante acentuados nas encostas da serra, sobretudo do lado sul. Ressalta-se que na área dos campos de altitude a Ts atingiu $36,6^{\circ} \mathrm{C}$, temperatura esta igual à observada em baixas elevações evidenciando os efeitos de diferentes superfícies de materiais sobre a serra, que emitem a radiação de ondas longas, diferentemente. Dozier \& Outcalt (1979) observaram que os gradientes termais coincidem com as transições de tipos de superfícies materiais.

Outro fato passível de ter contribuído para a redução da Ts com a altitude, pode estar relacionado com a precipitação pluvial e rarefação do ar. Silva et al. (2011) observaram diminuição da Ts quando aplicada irrigação em pomar de coqueiros no Nordeste brasileiro. Em agosto, Figura 4B, a Ts oscilou entre 15,5 a $33,7^{\circ} \mathrm{C}$ com amplitude térmica de 18,2 ${ }^{\circ} \mathrm{C}$. Observa-se, ainda, diminuição na Ts à medida em que a elevação aumenta nas encostas voltadas para o sul porquanto há um aumento da Ts com a elevação nas voltadas para o norte. Este aumento está relacionado com os efeitos de inclinação e com a exposição do terreno em relação à posição do Sol que ilumina as encostas norte da serra com mais intensidade do que as encostas sul em agosto. Dozier \& Outcalt (1979) afirmam 
A.

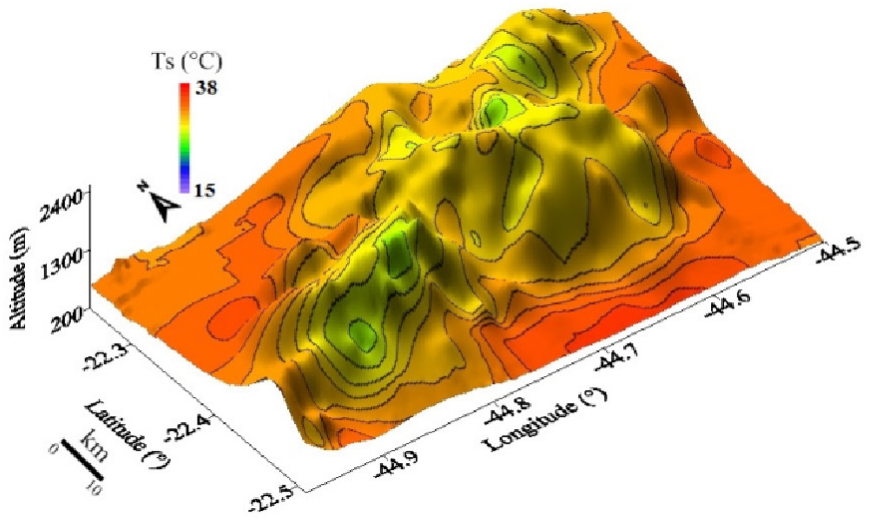

B.

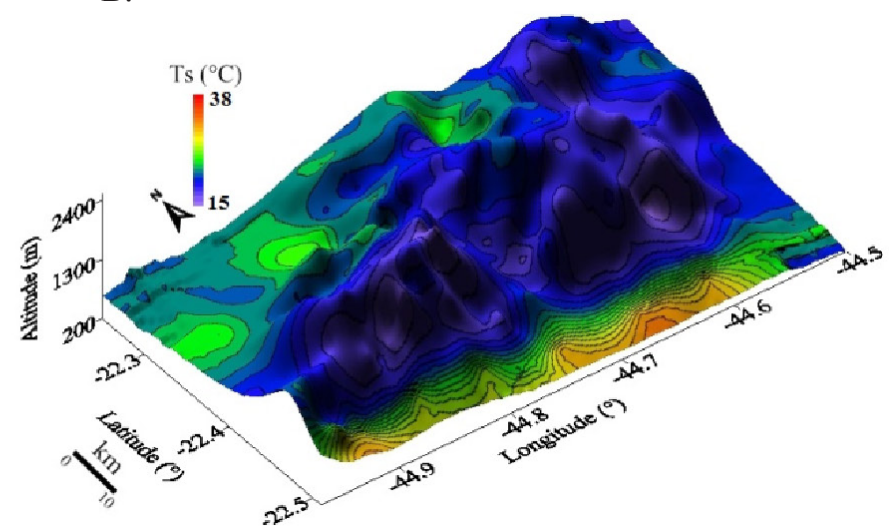

Figura 4. Temperatura média da superfície para fevereiro (A) e para agosto (B) de 2003 interpolados para 349 pixels extraídos dos produtos MOD43 e MYD11 às 10 h 30 min (hora solar) sobre a Serra da Mantiqueira

que esses efeitos produzem pequenas diferenças na Ts; contudo, se Ts for modelada em região de topografia bastante acentuada e de vegetação densa tornar-se-á necessário modelar o fluxo e a drenagem de ar, a atenuação do dossel e os processos convectivos locais.

A Figura 5A e a Figura 5B mostram a distribuição da radiação global solar (Rs) para os meses de fevereiro e agosto de 2003, respectivamente. Em fevereiro, Figura 5A, Rs varia entre 25,5 a 27,4 $\mathrm{MJ} \mathrm{m}^{-2}$, com isolinhas espaçadas $0,1 \mathrm{MJ} \mathrm{m}^{-2}$. Observa-se que a Rs aumenta com a altitude devido ao aumento da radiação solar direta e a diminuição da radiação solar difusa. Os efeitos de exposição e de inclinação do terreno podem ser vistos nesta figura. As encostas voltadas para o sul são menos iluminadas que as voltadas para o norte. As isolinhas de Rs são mais próximas nas encostas voltadas para o sul indicando maior variação de Rs, acentuado-se com a elevação. Em agosto, Figura 5B, Rs oscila entre 14,9 e 23,2 $\mathrm{MJ} \mathrm{m}^{-2}$, diminuindo bastante com a altitude. Este aumento na amplitude de Rs está associado principalmente à diminuição da incidência solar no inverno e à presença de cobertura de nuvens. Lopes \& Valeriano (2009) têm observado, na maior parte dos dias de inverno (agosto) céu aberto sobre o Vale do Rio Paraíba do Sul e cobertura de nuvens sobre a Serra da Mantiqueira. Oliphant et al. (2003) afirmaram que em dias de céu aberto a topografia é o principal modelador da radiação solar global; em dias de céu nublado as nuvens atenuam a entrada de Rs, sobremaneira nos locais de maiores

A.

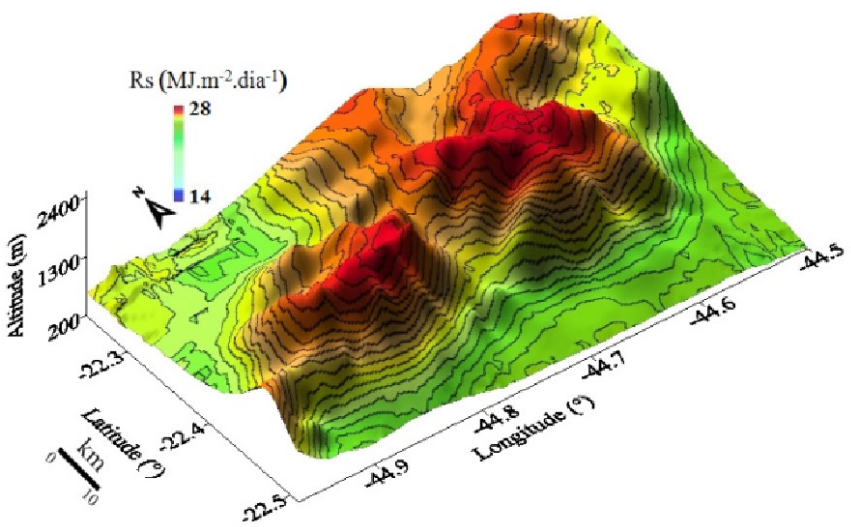

altitudes. Esta diferença de radiação solar global é percebida nas encostas mais íngremes da serra que sofrem grandes variações sazonais em sua iluminação. Lopes (2006) incluiu a presença da cobertura de nuvens na modelagem de radiação solar global na Serra da Mantiqueira observando uma redução no intervalo de incidência de radiação solar de aproximadamente $55 \%$. Esta redução em Rs pode chegar a até $100 \%$ em dias de céu encoberto, dependendo do local considerado.

A distribuição espacial da temperatura média do ar ( $\mathrm{t}$ ) para os meses de fevereiro e agosto de 2003 é mostrada na Figura 6A e na Figura 6B; em fevereiro, Figura 6A, a menor $\mathrm{t}$ foi de $21,1^{\circ} \mathrm{C}$ a $2555,8 \mathrm{~m}$ de altitude e a maior de $30,1^{\circ} \mathrm{C}$ em 460,4 $\mathrm{m}$ de altitude, com a média da área de $25,7^{\circ} \mathrm{C}$; já para o mês de agosto, Figura $6 \mathrm{~B}$, a t variou entre $12,5^{\circ} \mathrm{C}$ a $2555,8 \mathrm{~m}$ e $21,6^{\circ} \mathrm{C}$ a $460,4 \mathrm{~m}$ de altitude, apresentando temperatura média da área de $17,1^{\circ} \mathrm{C}$. Observa-se, ainda, que o lado sul da serra experimenta as maiores $t$ devido o vale do Rio Paraíba do Sul apresentar pastos e áreas urbanas e estar localizado entre a Serra da Mantiqueira mais elevada e a Serra do Mar, experimentando um clima quente e úmido no verão e frio e seco no inverno. Bardin et al. (2010) observaram que o fator altitude é o de maior influência na estimativa da $t$, pois para uma variação de $100 \mathrm{~m}$ de altitude a temperatura mínima varia entre 0,6 e 0,8 ${ }^{\circ} \mathrm{C}$. Lopes et al. (2011) observaram que, à medida em que se afastam da linha do equador em direção ao Sul do Brasil, as altitudes aumentam, diminuindo a t.

B.

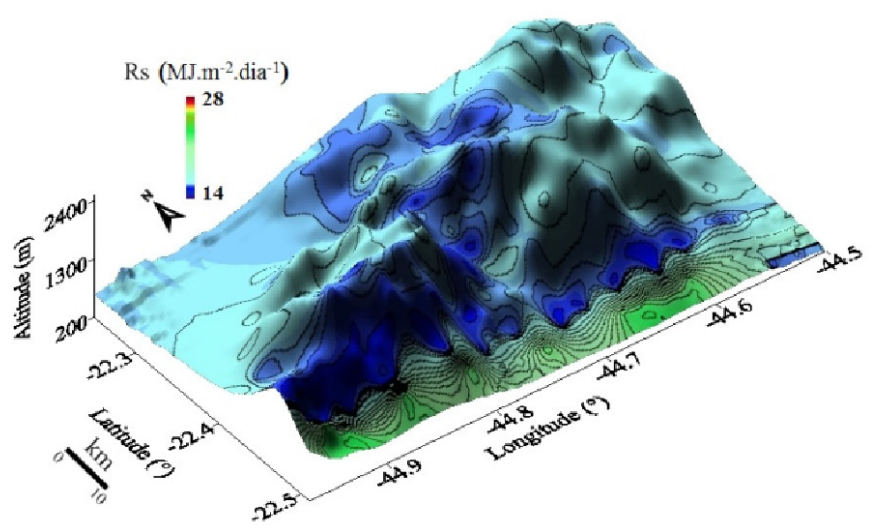

Figura 5. Radiação solar global para fevereiro (A) e para agosto (B) de 2003 sobre a Serra da Mantiqueira 
A.

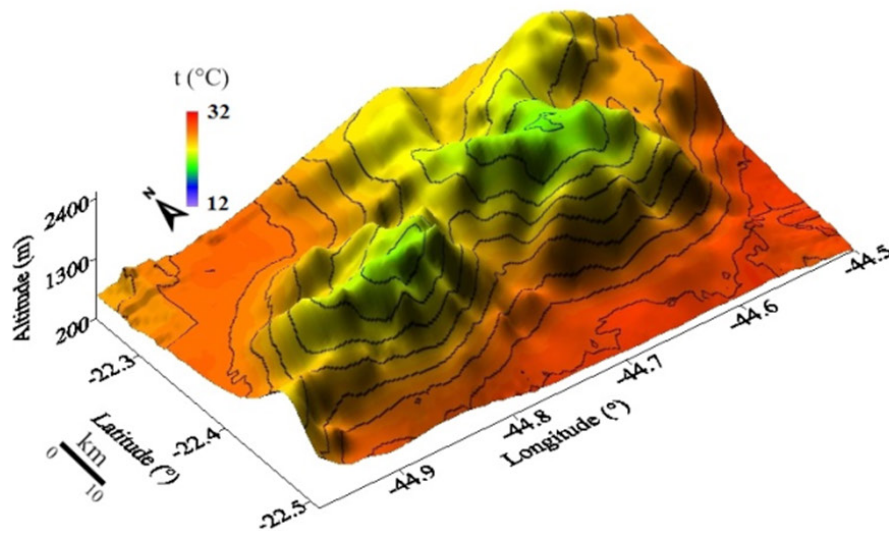

Figura 6. Temperatura média diária do ar para fevereiro

Estudos anteriores têm mostrado que, além da altitude, as características do relevo (Hungerford et al., 1989), a influência da temperatura da superfície do mar (Cavalcanti et al., 2006), as passagens de massas de ar frio sobre as Regiões Sul e Sudeste do Brasil, o resfriamento da superfície terrestre acentuado devido à estação de inverno proporcionando dias de céu aberto sobre o vale e a formação de nuvens orográficas sobre a serra (Lopes \& Valeriano, 2009) entre outros, influenciam as estimativas da t.

As distribuições espaciais do saldo de radiação à superfície (Rn) são mostradas nas Figura 7A e 7B, para fevereiro e agosto de 2003, respectivamente. Observa-se, na Figura 6A, que Rn é menor sobre o vale $\left(8,3 \mathrm{MJ} \mathrm{m}^{-2}\right)$, aumentando com a elevação do terreno, chegando a $13,8 \mathrm{MJ} \mathrm{m}^{-2}$ na serra. Em agosto, Figura $7 \mathrm{~B}, \mathrm{Rn}$ tem comportamento similar ao de fevereiro porém os valores são bem menores. A baixas altitudes, o saldo de radiação atinge valores de até 7,8 $\mathrm{MJ} \mathrm{m}^{-2}$, enquanto sobre a serra $\mathrm{Rn}$ chega a valores próximos de $2,5 \mathrm{MJ} \mathrm{m}^{-2}$. Para Oliphant et al. (2003) a diminuição em Rn está relacionada a fatores locais, tais como o aspecto, a cobertura de nuvens, a persistência de nevoeiros, os gradientes verticais de temperatura do ar e o índice de área foliar. Gomes et al. (2009) observaram, na região dos Cerrados, que aumentos na temperatura da superfície fazem diminuir Rn. Esses autores citam, como fontes de variação de $\mathrm{Rn}$, a quantidade de radiação de onda longa incidente na área de estudo que em fevereiro é substancialmente superior aos valores que ocorrem no mês de agosto e também na resposta da vegetação à sua fenologia e distribuição intra-anual das

A.

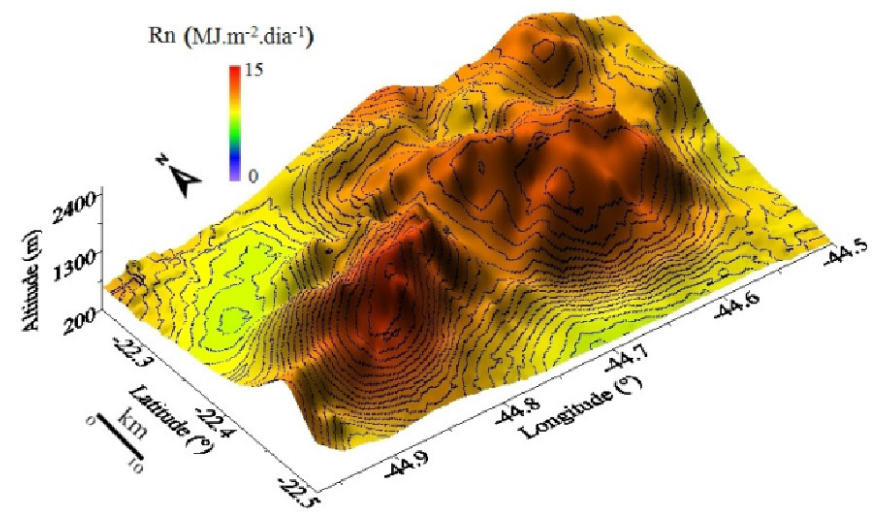

B.

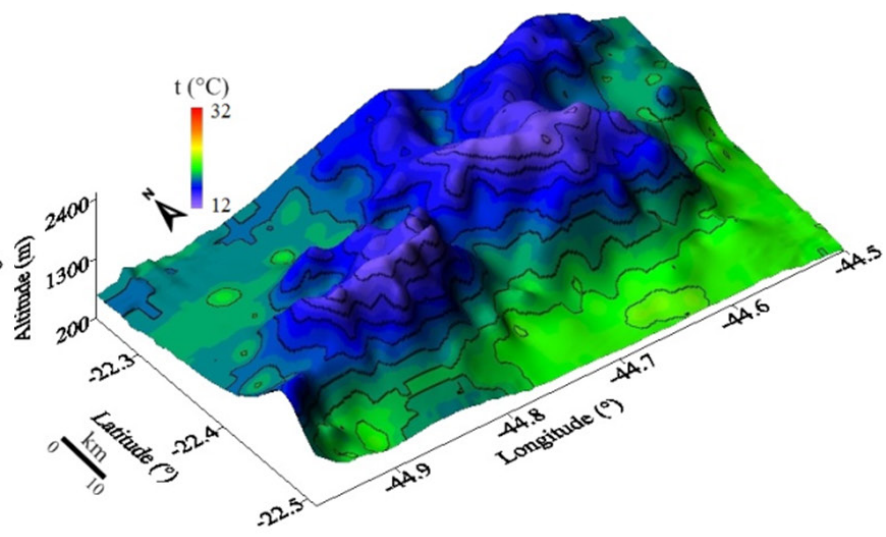

(A) e para agosto (B) de 2003 sobre a Serra da Mantiqueira

precipitações pluviais mensais. Nas áreas nas quais os valores de Rn são elevados há possibilidade de que a evapotranspiração também seja elevada. Fietz \& Fisch (2009) advertem que isto só é possível de acontecer se não houver restrições hídrica na área de estudo.

$\mathrm{Na}$ Tabela 2 estão representados alguns elementos meteorológicos e componentes do balanço de radiação para cinco diferentes tipos de cobertura do solo selecionados de acordo com a classificação não supervionada (Figura 1), a saber: floresta primária (FP), floresta secundária (FS), campos de altitude (CA), pastagem (P) e área de reflorestamento (AR). Em fevereiro o menor valor de Rn médio foi de $10 \mathrm{MJ} \mathrm{m}^{-2} \mathrm{em}$ áreas de $\mathrm{P}$ (altitude média $734 \mathrm{~m}$ ) e o maior $13 \mathrm{MJ} \mathrm{m}^{-2} \mathrm{em} \mathrm{CA}$ (altitude média $2206 \mathrm{~m}$ ). Percebe-se que baixos valores de Rn em $\mathrm{P}$ estão associados a valores relativamente pequenos de $\alpha$ $(16 \%)$ devido a cobertura vegetal não estar cobrindo totalmente o solo $(\mathrm{Pv}=59 \%)$ que favorece o aumento na absorção de Rs pelo solo em baixas altitudes elevando Ts $\left(34{ }^{\circ} \mathrm{C}\right)$ e a saída de $R_{\mathrm{L} \uparrow}\left(-41 \mathrm{MJ} \mathrm{m}^{-2}\right)$ em comparação com a entrada de $\mathrm{R}_{\mathrm{L} \downarrow}$ $\left(30 \mathrm{MJ} \mathrm{m}^{-2}\right)$. A diminuição ainda maior em $\alpha(12 \%)$ sobre $\mathrm{CA}$ está associada à redução da cobertura vegetal $(\mathrm{Pv}=29 \%)$ que contribui para aumentar o balanço de ondas curtas e à altitude, que reduz Ts $\left(29^{\circ} \mathrm{C}\right)$ e t $\left(23^{\circ} \mathrm{C}\right)$ aumentado $\mathrm{Rn}$. Em agosto a passagem de frentes frias contribui para diminui Rn sobre toda a área, sendo mais acentuada em P, FS e CA.

A Ts tem contribuído para aumentar Rn, implicando em maior aquecimento do ar sobre $\mathrm{P}$ do que sobre FP e FS, o que

B.

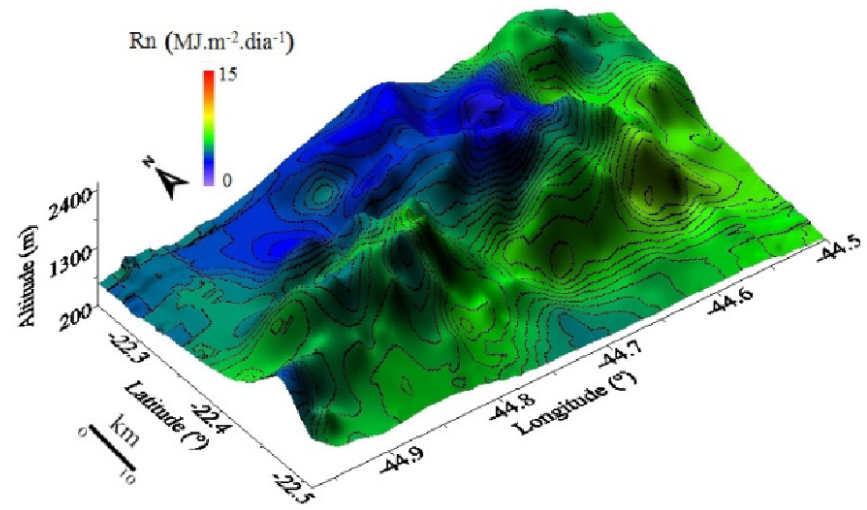

Figura 7. Saldo de radiação solar à superfície para fevereiro (A) e para agosto (B) de 2003 sobre a Serra da Mantiqueira 
Tabela 2. Intervalos e valores médios de albedo $(\alpha)$ e da temperatura da superfície (Ts), extraídos dos produtos MOD11 e MOD43, temperatura do ar (t) estimada pelo modelo MTCLIM, radiação de ondas longas da superfície $\left(\mathrm{R}_{\mathrm{L},}, 24 \mathrm{~h}\right)$ e da atmosfera $\left(\mathrm{R}_{\mathrm{L},}, 24 \mathrm{~h}\right)$ e balanço de radiação na superfície $(\mathrm{Rn}, 24 \mathrm{~h}),{ }^{*}(\mathrm{)})$ valores médios das variáveis

\begin{tabular}{|c|c|c|c|c|c|c|c|}
\hline Uso da terra & Meses & $\underset{(\%)}{\alpha}$ & $\begin{array}{l}\text { Ts } \\
\left({ }^{\circ} \mathrm{C}\right)\end{array}$ & $\begin{array}{c}t \\
\left({ }^{\circ} \mathrm{C}\right)\end{array}$ & $\begin{array}{l}R_{\mathrm{L} \uparrow}, 24 \mathrm{~h} \\
\left(\mathrm{MJ} \mathrm{m}^{-2}\right)\end{array}$ & $\begin{array}{l}R_{\mathrm{Ll}}, 24 \mathbf{h} \\
\left(\mathrm{MJ} \mathrm{m}^{-2}\right)\end{array}$ & $\begin{array}{c}\operatorname{Rn} 24 \mathrm{~h} \\
\left(\mathrm{MJ} \mathrm{m}^{-2}\right)\end{array}$ \\
\hline Floresta primária & Fevereiro & $10-17(13)^{*}$ & $28-35(31)$ & $22-29(26)$ & $-42-31(-38)$ & $21-30(27)$ & $8-15(12)$ \\
\hline (FP) & Agosto & $8-16(12)$ & $16-28(19)$ & $14-21(17)$ & $-23-38(-32)$ & $18-27(24)$ & $3-9(6)$ \\
\hline Floresta secundária & Fevereiro & $12-16(15)$ & $29-36(32)$ & $24-30(28)$ & $-43-34(-39)$ & $24-31(28)$ & $8-13(11)$ \\
\hline (FS) & Agosto & $9-16(13)$ & $18-33(23)$ & $16-21(19)$ & $-40-26(34)$ & $19-27(25)$ & $3-8(5)$ \\
\hline Campos de altitudes & Fevereiro & 8-14 (12) & $27-32(29)$ & $21-25(23)$ & $-39-31(-34)$ & $22-28(24)$ & $11-15(13)$ \\
\hline$(\mathrm{CA})$ & Agosto & $8-14(11)$ & $16-19(17)$ & $13-16(14)$ & $-32-27(-30)$ & $20-24(22)$ & 4-7 (5) \\
\hline Pastagem & Fevereiro & $13-18(16)$ & $31-36(34)$ & $26-30(29)$ & $-43-33(-41)$ & $23-31(30)$ & $8-11(9)$ \\
\hline$(\mathrm{P})$ & Agosto & 11-17 (14) & $19-33(26)$ & $17-22(20)$ & $-41-24(-35)$ & $18-27(26)$ & $3-7(5)$ \\
\hline Area de reflorestamento & Fevereiro & $14-16$ (15) & $30-36(33)$ & $27-30(29)$ & $-42-38(-40)$ & $29-31(30)$ & $9-12(10)$ \\
\hline (AR) & Agosto & $14-16(15)$ & $19-31(25)$ & $19-22(20)$ & $-39-31(-35)$ & $25-27(26)$ & $5-6(6)$ \\
\hline
\end{tabular}

está de acordo com Gomes et al. (2009) que estimaram o Rn com o SEBAL/METRIC na bacia do Mogi-Guaçu, São Paulo, concluindo que a substituição de vegetação primária por áreas agrícolas e pastagem tem contribuído para aumentar a Ts e diminuído Rn. Silva et al. (2011) observaram, no Perímetro Irrigado São Gonçalo, na Paraíba e em áreas adjacentes, valores pequenos de Rn em afloramento rochoso e em áreas com grande exposição do solo. Em geral, valores menores de $\alpha$ e maiores de Ts, tême menor disponibilidade de energia contribuindo para deixar as áreas de pastos mais quentes que sua vizinhança local, em fevereiro e agosto de 2003.

\section{Conclusões}

1. O modelo MTCLIM tem demonstrado boa precisão na estimativa da temperatura do ar $\left(\mathrm{R}^{2}=0,82\right)$ e da radiação solar global $\left(\mathrm{R}^{2}=0,71\right)$ e pode ser usado para estimar o balanço de energia em regiões montanhosas.

2. O balanço de radiação à superfície acompanhou o relevo local e é fortemente influenciado pelo tipo de uso da terra.

3. Em áreas de floresta e de campos de altitudes o balanço de radiação à superfície foi maior implicando em menor aquecimento do ar e da superfície, disponibilizando mais energia para os processos de evapotranspiração, condensação e fotossíntese.

4. Em pastos e área de reflorestamento a disponibilidade de energia foi menor, contribuindo para deixar essas áreas mais quentes que a sua vizinhança local.

\section{Agradecimentos}

À CAPES, pela concessão de bolsa de Pós-Graduação ao primeiro autor durante o estudo; ao CPTEC, pela concessão dos dados meteorológicos; à LPDAAC/NASA, pela concessão de dados orbitais e ao Corpo Editorial, pelo valioso trabalho e contribuições.

\section{Literatura Citada}

Allen, R.; Tasumi, M.; Trezza. R. Satellite-based energy balance for mapping evapotranspiration with internalized calibration (METRIC)-Model. Journal of Irrigation and Drainage, E-ASCE, v.133, p.380-394, 2007.
Allen, R. G.; Trezza, R.; Tasumi, M. Surface energy balance algorithm for land. Advance training and user's manual, version 1.0. Kimberly: University of Idaho, 2002. 98p.

Almeida, A. C; Landsberg, J. J. Evaluating methods of estimating global radiation and vapor pressure deficit using a dense network of automatic weather stations in coastal Brazil. Agricultural and Forest Meteorology, v.118, p.237250, 2003.

Bardin, L.; Pedro Júnior, M. J.; de Moraes, J. F. L. Estimativa das temperaturas máximas e mínimas do ar para a região do Circuito das Frutas, SP. Revista Brasileira de Engenharia Agrícola e Ambiental, v.14, p.618-624, 2010.

Borges, V. P.; Oliveira, A. S.; Coelho Filho, M. A.; Silva, T. S. M. da; Pamponet, B. M. Avaliação de modelos de estimativa da radiação solar incidente em Cruz das Almas, Bahia. Revista Brasileira de Engenharia Agrícola e Ambiental, v.14, p.74-80, 2010.

Bristow, K.; Campbell, G. On the relationship between incoming solar rariation and daily maximum and minimum temperature. Agricultural and Forest Meteorology, v.31, p.159-166, 1984.

Cavalcanti, E. P.; Silva, V. de P. R. da; Souza, F. de A. S. de. Programa computacional para a estimativa da temperatura do ar para a região Nordeste do Brasil. Revista Brasileira de Engenharia Agrícola e Ambiental, v.10, p.140-147, 2006.

Di Pace, F. T.; Silva, B. B. da; Silva, V. de P. R. da; Silva, S. T. A. Mapeamento do saldo de radiação com imagens Landsat 5 e modelo de elevação digital. Revista Brasileira de Engenharia Agrícola e Ambiental, v.12, p.385-392, 2008.

Dozier, J.; Outcalt, S. I. An approach toward energy balance simulation over rugged terrain. Geographycal Analysis, v.11, p.65-85, 1979.

Fietz, C. R.; Fisch, G. F. Avaliação de modelos de estimativa do saldo de radiação e do método de Priestley-Taylor para a região de Dourados, MS. Revista Brasileira de Engenharia Agrícola e Ambiental, v.13, p.449-453, 2009.

Glassy, J. M.; Running, S.W. Validating diurnal climatology logic of the MT-CLIM model across a climatic gradient in Oregon. Ecological Applications, v.4, p.248-257, 1994.

Gomes, H. B.; Silva, B. B. da; Cavalcanti, E. P.; Rocha, H. R. Balanço de radiação em diferentes biomas no estado de São Paulo mediante imagens Landsat 5. Geociências, v.28, p.153-164, 2009. 
Hungerford, R. D.; Nemani, R. R.; Running, S.; Coughlan, J. C. MTCLIM: A mountain microclimate simulation model. Ogden: USDA: Intermountain Research Station, Forest Service, 1989. 51p.

Lopes, P. M. O. Obtenção de regimes microclimáticos em regiões montanhosas com dados de sensores orbitais e integração de modelos distribuídos. São José dos Campos: INPE, 2006. 229p. Tese Doutorado

Lopes, P. M. O.; Adami, M.; Pereira, E. B.; Moura, G. B. de A.; Moreira, M. A. Estimativa de temperaturas absolutas usando dados climáticos e orbitais sobre o território brasileiro. Revista Caatinga, v.24, p.120-127, 2011.

Lopes, P. M. O.; Valeriano, D. de M. Regimes de temperatura do ar em região montanhosa. Revista Geográfica Acadêmica, v.3, p.54-67, 2009.

LPDAAC - Land Processes Distributed Actives Archive Center. $<$ lpdaac.usgs.gov/products/modis_products_table>,9Mai. 2012.

Morellato, L. P. C.; Haddad, C. F. B. Introduction: The Brazilian Atlantic Forest. Biotropica, v.32, p.786-792, 2000.

Oliphant, A. J.; Smith, R. A. S.; Sturman, A. P.; Owens, I. F. Spatial variability of surface radiation fluxes in mountainous terrain. Journal of Applied Meteorology, v.42, p.113-128, 2003.

Running, S. W.; Nemani, R. R.; Hungerfored, D. R. Extrapolation of synoptic meteorological data in mountainous terrain and its use for simulating FOREST evapoTrapiration and photosynthesis, Canadian Journal of Forest Resource, v.17, p.472-483, 1987.

Salisbury, J. W.; D’Aria, D. M. Emissivity of terrestrial material in the $8-14 \mu \mathrm{m}$ atmospheric window. Remote Sensing of Environment, v.42, p.83-106, 1992.

Santos, C. A. C.; Silva, B. B. da. Obtenção dos fluxos de energia à superfície utilizando o algoritmo S-SEBI. Revista Brasileira de Meteorologia, v.25, p.365-374, 2010.
São Thiago, L. E. U.; Barros, M. A.; Barth, O. M. Pollen deposition in soil sediments from the middle valley of Paraíba do Sul river (São Paulo and Rio de Janeiro States). Anuário do Instituto de Geociências, v.30, p.5564, 2007.

Silva, B. B. da; Braga, A. C.; Braga, C. C. Balanço de radiação no perímetro irrigado São Gonçalo - PB mediante imagens orbitais. Revista Caatinga, v.24, p.145-152, 2011.

Silva, B. B.; Lopes, G. M.; Azevedo, P. V. Balanço de radiação em áreas irrigadas utilizando imagens Landsat 5 - TM. Revista Brasileira de Meteorologia, v.20, p.243$252,2005$.

Silva, V. de P. R. da; Azevedo, P. V. de; Silva, B. B. da. Surface energy fluxes and evapotranspiration of a mango orchard grown in a semiarid environment. Agronomy Journal, v. 99, p.1391-1396, 2007.

Thornton, P. E.; Hasenauer, H.; White, M. A. Simultaneous estimation of daily solar radiation and humidity from observed temperature and precipitation: An application over complex terrain in Austria, Agricultural and Forest Meteorology, v.104, p.255-271, 2000.

Thornton, P. E.; Running, S. W. An improved algorithm for estimating incident daily solar radiation from measurements of temperature, humidity, and precipitation. Agricultural and Forest Meteorology, v.93, p.211-228, 1999.

Valor, E.; Caselles, V. Mapping land surface emissivity from NDVI: Application to European, African, and South American Areas. Remote Sensing of Environment, v.57, p.167-18, 1996.

Xiong, X.; Chiang, K.; Sun, J.; Barnes, W. L.; Guenther, B.; Salomonson, V. V. NASA EOS Terra and Aqua MODIS on-orbit performance. Advances in Space Research, v.43, p.413-422, 2009. 